\title{
The Characteristics of Global Shallow-Source Seismicities Associated with Solar Activities in Different Time Scales
}

\author{
Xiao. Xia. Yu ${ }^{1,2}$ \\ 1.Key Laboratory of Particle Astrophysics, Institute of High Energy Physics, Chinese Academy of \\ Sciences \\ 19B Yuquan Road, Shijingshan District, Beijing, China \\ 2. State Key Laboratory of Space Weather, Chinese Academy of Sciences, Beijing, China \\ E-mail: xxyu@ihep.ac.cn
}

\section{Chun Lan Jin ${ }^{3}$}

3.Key Laboratory of Solar Activity, National Astronomical Observatories, Chinese Academy of Sciences, Beijing, China

E-mail:cljin@nao.cas.cn

\section{Zheng. Hua. $\mathrm{An}^{1}$}

1.Key Laboratory of Particle Astrophysics, Institute of High Energy Physics, Chinese Academy of Sciences

19B Yuquan Road, Shijingshan District, Beijing, China

E-mail: anzh@ihep.ac.cn

\begin{abstract}
Here we report the characteristics of global shallow-source seismicities associated with solar activities in different time scales during the past 10 solar cycle periods (1902-2008). By using LombScargle spectrum analyses, about 11 years period exists in the number of earthquakes $(4 \leq \mathrm{M}<5$, about 190,000 earthquakes). In decadal time scale, higher number and released energy from earthquakes occurred around descending phases than ascending phases of solar cycle. Furthermore, higher number and more energy from earthquakes occurred around one year before and after minimum year $(\mathrm{m} \pm 1)$ and maximum year $(\mathrm{M} \pm 1)$ of sunspots number.
\end{abstract}

Keywords: Seismicity, solar activity, Lomb-Scargle algorithm

35th International Cosmic Ray Conference - ICRC2017

10-20 July, 2017

Bexco, Busan, Korea

\footnotetext{
${ }^{1}$ Speaker

(C) Copyright owned by the author(s) under the terms of the Creative Commons 
The Characteristics of Global Shallow-Source Seismicities Associated with Solar Activities in Different Time Scales.

\section{Introduction}

In the field of earthquake's long-term monitoring, the characteristics of earthquakes associated with solar activities is one of important subjects. Earthquakes and violent solar activities, such as solar flares, Coronal Mass Ejections (CMEs) and filaments, are both processes involving huge and rapid energy released. Recent studies have shown that the stochastic processes underlying apparently different phenomena such as solar flares and earthquakes have universal properties (Arcangelis, 2008; Arcangelis, 2006; Balasis, 2011). Odintsov et al. (2006) discovered that there is connection between solar activity and seismicity on both secular (Gleissberg) and decadal (11-year solar cycle) time-scales. Shaltout et al. (1999) investigated the autocorrelation and power spectra analyses on the sequences of sunspots and earthquakes. They found that there are close relations between sunspot periodicities and seismicities on the world-wide scale, or on the North African scale. In their opinion, the mechanism of the influence of solar activity on seismicity is one of the seven possibilities, such as variations in the Earth's atmospheric processes or variations in the solar wind parameters (Shaltout, 1999), etc. In addition, Simpson (1967) suggested that terrestrial solar flare effects which are actual coupling mechanisms which trigger quakes appear to be either abrupt acceleration in the earth's angular velocity or surge of telluric currents in the earth's crust. Furthermore, Gousheva et al. (2003) proposed that an obvious enhancement of earthquakes occurrence rate happened on the arrival day of high-speed solar wind and one day after it (Gousheva, 2003; Marilia, 2011).

In addition, the existence of electrical and magnetic disturbances associated with earthquakes has long been observed. Demeter satellite, as a detection of electro-magnetic emissions transmitted from earthquake regions, has been used to study disturbances in the upper atmosphere related to natural geophysical phenomena, such as earthquakes, volcanic eruptions or tsunamis (tidal waves). Electromagnetic emissions from the ground, electromagnetic emissions, ionosphere disturbances have been detected in several earthquake cases. It is interesting for us to investigate the Sun-Earth coupling system that includes ground, atmosphere, ionosphere and solar wind environment for the earthquake prediction (Inan, 2007).

In the paper, the characteristics of global shallow-source seismicities associated with solar activities in different time scales (i.e. decadal scale, one-year scale and one-day scale) during the past 10 solar cycle periods (1902-2008) are revealed. Section 2 will restrict our attention to the number $(\mathrm{N})$, released energy $(\mathrm{E})$ from the earthquakes and solar activity index, based on the global 251,107 shallow-source earthquakes $(4.0 \leqslant$ magnitude $<10.0$, focal depth $\leqslant 100 \mathrm{~km}$ ), the smoothed sunspot numbers (R), Sun-Earth relative position, and local time of epicenter. Section 3 will be devoted to the discussions of our results and further speculations.

\section{Data Analyses}

Since solar activity affects the earth globally, the analysis of seismic events should be on a global scale rather than focusing on a certain region (Marilia 2011, Yu 2010). To some extent, aftershocks cover up the statistical characteristics of the earthquake as individual events, so we select independent earthquakes to reduce occurrence rates of the earthquakes near source area from the aftershocks. In our paper, the focal depth for the selected shallow-source earthquakes are less than $100 \mathrm{~km}$ during the past 10 solar cycle periods (1902-2008), because the crust can be affected by solar activities to some extent (Jusoh, 2011). In Figure 1, we describe the 
The Characteristics of Global Shallow-Source Seismicities Associated with Solar Activities in Different Time Scales.

geographical distribution map and focal depth of global 4457 shallow-source earthquakes $(6 \leqslant$ $\mathrm{M}<10$ ), and the rest of earthquakes are omitted due to the clarity of the figure. The global 251,107 shallow-source earthquakes cases $(4 \leqslant \mathrm{M}<10)$ are listed in supplementary Table 1 .

Table 1. The global 251,107 shallow-source earthquakes cases $(4 \leqslant M<10)$ are listed.

\begin{tabular}{|c|c|c|c|c|c|}
\hline Depth $\backslash$ Magnitude & $\mathbf{8} \leq \mathbf{M}<\mathbf{1 0}$ & $\mathbf{7} \leq \mathbf{M}<\mathbf{8}$ & $\mathbf{6} \leq \mathbf{M}<\mathbf{7}$ & $\mathbf{5} \leq \mathbf{M}<\mathbf{6}$ & $\mathbf{4} \leq \mathbf{M}<\mathbf{5}$ \\
\hline$\leq 25 \mathrm{Km}$ & 14 & 199 & 1730 & 14239 & 59723 \\
\hline$\leq 50 \mathrm{Km}$ & 29 & 427 & 3473 & 43267 & 162216 \\
\hline$\leq 100 \mathrm{Km}$ & 32 & 469 & 3956 & 53061 & 193589 \\
\hline
\end{tabular}

The formula of energy released in earthquakes is calculated from the following expression (Kanamori, 1977):

$$
\log E=11.8+1.5 \mathrm{M}
$$

where $\mathrm{E}$ is expressed in erg units. The data of global 251,107 shallow-source earthquakes $(6 \leqslant M<10)$, focal depth $\leqslant 100 \mathrm{~km})$, and the smoothed sunspot numbers (R) were taken from the scientific data web sites (http://www.ncedc.org/anss/catalog-search.html, http://www.sws.bom.gov.au/Educational/2/3/6).

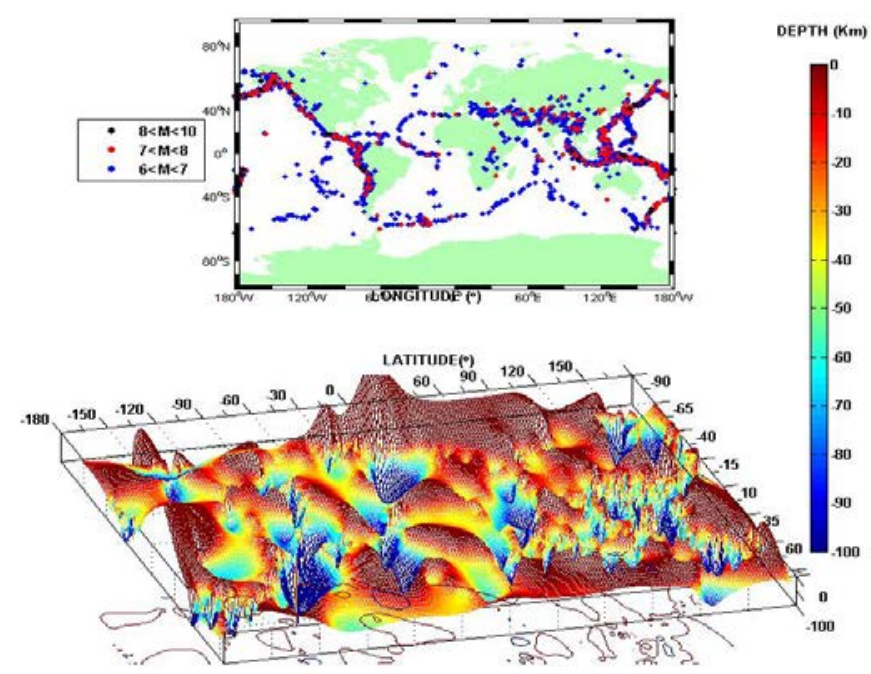

Figure 1. Geographical distribution map and focal depth of the global 4457 shallow-source earthquakes $(6 \leq \mathrm{M}<10$, focal depth $\leq 100$ $\mathrm{km}$ ) for the period 1902-2008. Top panel: The geographical distribution of earthquakes. Black circles, red circles and blue circles denote earthquakes with magnitude $8.0 \sim 10.0$, 7.0 8.0, 6.0 7.0, respectively. Bottom panel: Contour maps of focal depth for the earthquakes. The global 251,107 shallow-source earthquakes cases $(4 \leq \mathrm{M}<10)$ are listed in Table 1.

\subsection{Century Scale}

Earthquakes and solar activities, such as solar flares, CMEs, filaments, manifest highly energetic explosions from solar active regions in the form of electromagnetic radiation, particle and plasma flows powered by strong and twisted magnetic fields. In solar activities, the century scale variations are called Gleissberg cycle (Gleissberg, 1939). Some analyses show that solar activity can heavily influence the Earth's magnetosphere, creating fluctuations which are known as geomagnetic storms. Sunspot numbers are a good indication of solar activity, and it may be better to look at something which is directly affected by solar eruptions and which in turn may influence earthquakes. Shaltout et al. (1999) have carried out power spectra analysis for North 
The Characteristics of Global Shallow-Source Seismicities Associated with Solar Activities in Different Time Scales.

Africa historical earthquakes data in relation to sunspots periodicities, and their results indicated a periodicities ranged between 1.01 and 5.5 years exited, which may be associated with a Solar Cycle.

The Fourier amplitude spectrum analysis is a powerful technique in searching for the periodic signal in time series, but the unevenly sampled time series may cause spurious contribution to the spectrum analysis. For this reason, an alternative method of time-series analysis has become increasingly popular in earth sciences and astronomy, called the LombScargle algorithm (Scargle, 1982; Li et al., 2008). The Lomb-Scargle algorithm evaluates the data of the time series only at times t $\mathrm{j}$ that are measured. A series $\mathrm{X}(\mathrm{t})$ with $\mathrm{N}$ data points is supposed, and the Lomb-Scargle normalized periodogram $\mathrm{PN}(\omega)$ as a function of angular frequency ( $\omega=2 \pi \mathrm{f}>0$ ) is given by

$$
P_{N}(\omega)=\frac{1}{2 \sigma^{2}}\left\{\frac{\left[\sum_{j}\left(X\left(t_{j}\right)-\bar{X}\right) \cos \omega\left(t_{j}-\tau\right)\right]^{2}}{\sum_{j} \cos ^{2} \omega\left(t_{j}-\tau\right)}+\frac{\left[\sum_{j}\left(X\left(t_{j}\right)-\bar{X}\right) \sin \omega\left(t_{j}-\tau\right)\right]^{2}}{\sum_{j} \sin ^{2} \omega\left(t_{j}-\tau\right)}\right\}
$$

Where

$$
\tan (2 \omega \tau)=\left(\sum_{j} \sin 2 \omega t_{j}\right) /\left(\sum_{j} \cos 2 \omega t_{j}\right)
$$

We can compute the falsealarm probability of the null hypothesis by $P(>z)=1-\left(1-e^{-z}\right)^{M}$.

Discontinuous acquisition of seismic data and poor detection precision of ground-based seismic monitoring instruments for $1902 \sim 1962$ let us carry out the spectrum analyses of the earthquakes for 1963 2008. In Figure 2, time series of sunspot numbers (1700 2014) are examined by different power spectral analysis methods (e.g. FFT algorithm and Lomb-Scargle algorithm). We find that about 11.05 years is the obvious periodicity for solar cycle in the two algorithms (the largest peak in Figure 2). The same period (about 11 years) also exits in the number of earthquakes $(4 \leqslant \mathrm{M}<5)$.
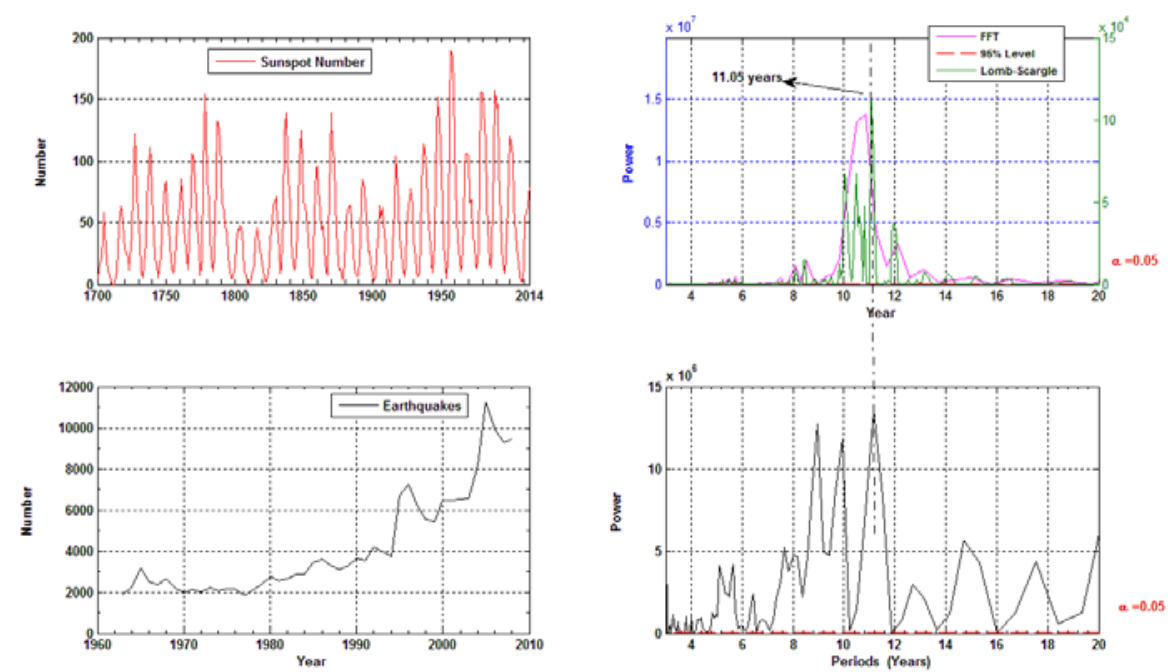

Figure 2. Power spectral analysis of sunspot number and earthquakes at century scale. Left panel: time sequence of sunspot number (1700 2014) and occurence rates of earthquakes (1963 2008, about 190,000 earthquakes cases). Right panel: spectrum analyses of sunspot number and earthquakes, and $\alpha=0.05$ means $95 \%$ significance level.

\subsection{Decadal Scale}


The Characteristics of Global Shallow-Source Seismicities Associated with Solar Activities in Different Time Scales.

The number of sunspots is a good indication of solar activity, so periodic change of sunspot number is usually used to differentiate solar cycle in the solar surface. In general, the solar cycle lasts for about 11 years (see Figure 2). It includes ascending phase, peak period and descending phase, of which the minimum and maximum of sunspot number can divide them clearly. We can determine the influences from different phases of solar cycle by subtracting the amount of number and energy of earthquakes happened in the ascending phase from the descending phase. If the difference is positive, it means higher frequency and more energy released in the descending phase of one solar cycle. In Figure 3 we infer that higher number of earthquakes occurred around descending phases of solar cycle than ascending phases. Released energy is the same. This conclusion is in accordance with Jusoh Mohamad's (2011) opinion, but our results are more accurate by eliminating the descending period longer than ascending period of one solar cycle. In order to verify the reliability of the above results, we select three different focal depths (i.e. $\leqslant 100 \mathrm{~km}, \leqslant 50 \mathrm{~km}, \leqslant 25 \mathrm{~km}$, respectively) and obtain the same conclusion.
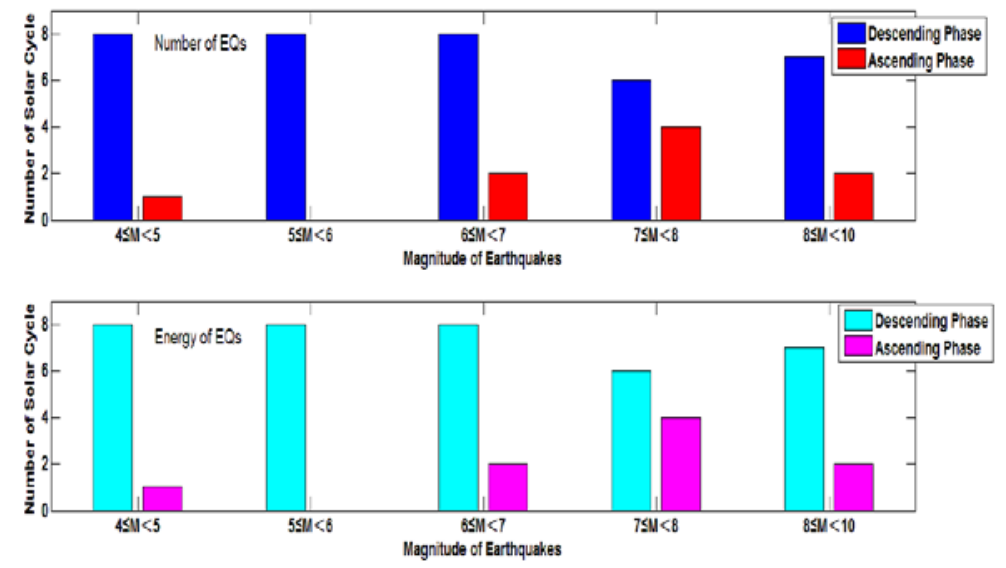

Figure 3. Higher earthquakes frequency and more energy released in the descending phase than the ascending phase of one solar cycle from the 14th to the 23rd solar cycle (1902-2008).

Top panel: blue bar means that difference of earthquakes number between descending phase and ascending phase in one solar cycle is $>0$, and red bar means that is $<0$.

Bottom column: green bar means that difference of energy from earthquakes between descending phase and ascending phase in one solar cycle is $>0$, and pink bar means that is $<0$. Y axes denotes the number of solar cycle. $\mathrm{X}$ axes indicates the magnitude of earthquakes.

In addition, the distributions of numbers and energy from earthquakes happened in one solar cycle are discussed below. We assume the annual earthquakes number and energy for the years from one year before and after minimum year $(\mathrm{m} \pm 1)$ and maximum year $(\mathrm{M} \pm 1)$ of sunspots number as $\mathrm{N} 1$ and $\mathrm{E} 1$, and assume the average earthquakes numbers and energy of one entire solar cycle as N2. If the difference between N1 and N2 is positive, it means that higher frequency and more energy for the years from one year before and after minimum year $(\mathrm{m} \pm 1)$ and maximum year $(\mathrm{M} \pm 1)$ of sunspots number. In Figure 4 we infer that higher number and released energy from earthquakes occurred around one year before and after minimum year $(\mathrm{m} \pm 1)$ and maximum year $(\mathrm{M} \pm 1)$ of sunspots number. The magnitude of an earthquake is higher, the more obvious trend. In order to verify the reliability of the above results, we select three different focal depths (i.e. $\leqslant 100 \mathrm{~km}, \leqslant 50 \mathrm{~km}, \leqslant 25 \mathrm{~km}$, respectively) and obtain the same conclusion. 
The Characteristics of Global Shallow-Source Seismicities Associated with Solar Activities in Different Time Scales.
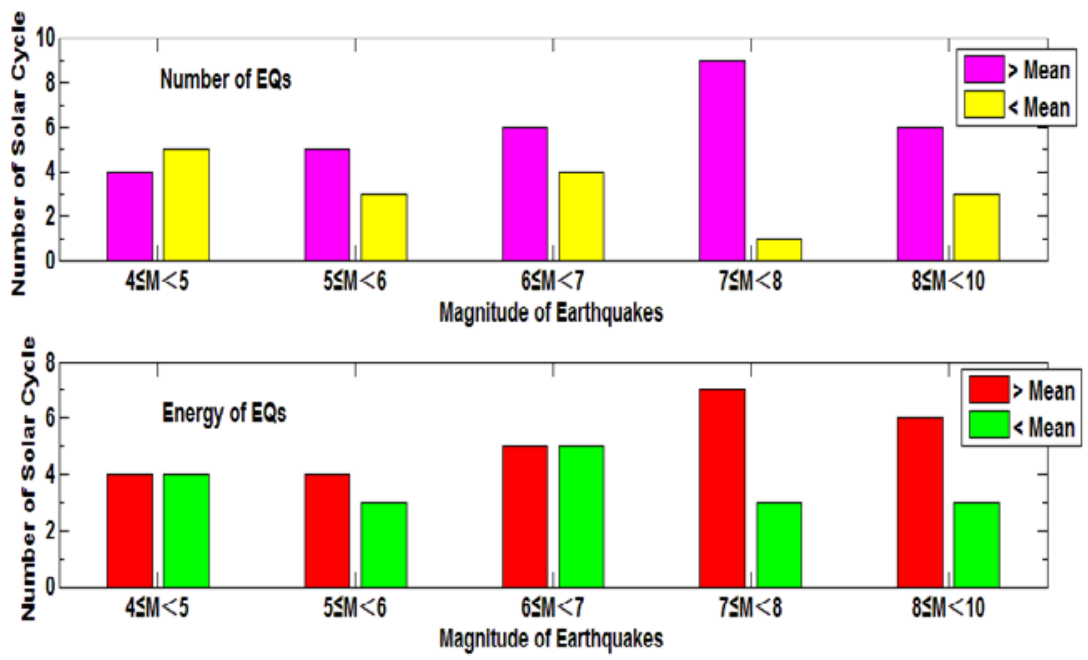

Figure 4. Higher frequency and more energy aroound one year before and after minimum year $(\mathrm{m} \pm 1)$ and maximum year $(\mathrm{M} \pm 1)$ of sunspots number. Top column: pink bar means number of earthquakes which occurred on minimum year $(\mathrm{m} \pm 1)$ and maximum year $(\mathrm{M} \pm 1)$ is larger than the average, and yellow bar means that is smaller than the average. Bottom column: read bar means that energy released on minimum year $(\mathrm{m} \pm 1)$ and maximum year $(\mathrm{M} \pm 1)$ is larger than the average, and green bar means that is smaller than the average. $\mathrm{Y}$ axes denotes the number of solar cycle. $\mathrm{X}$ axes indicates the magnitude of earthquakes.

\subsection{One-year Scale}

Considering the perihelion (January 1st to January 5th) and the aphelion (July 3rd to July 4 th), the relationship between occurrence frequency $(\mathrm{N})$, released energy $(\mathrm{E})$ and the relative Sun-Earth position are depicted in Figure 5. Inspection of the values in Figure 5 shows that occurrence rates of earthquakes have no significant difference in different months. But the number and energy from earthquakes $(4 \leqslant \mathrm{M}<6)$ in February is less than the other months. For large earthquakes $(8 \leqslant M<10)$ occurrence frequency $(N)$ are larger in March, August and October, and released energy (E) from earthquakes are more powerful in March and December. The energy released from shallow-source earthquakes with magnitude $(8 \sim 10)$ is dominant in the total seismic energy.
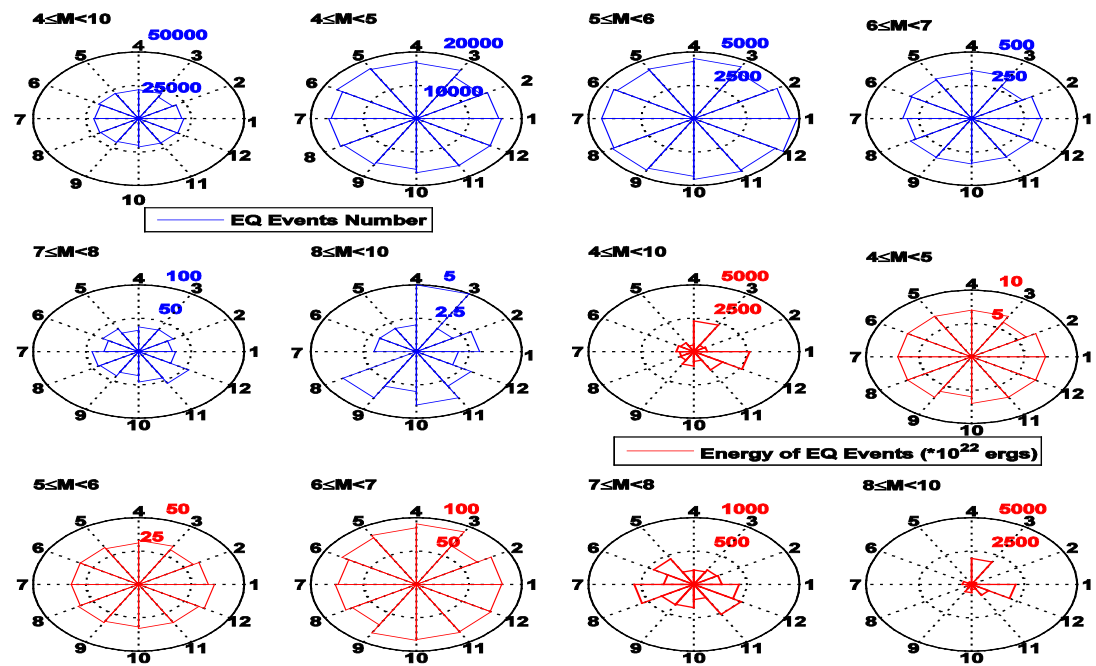

Figure 5. The relationship between occurrence frequency $(\mathrm{N})$, released energy $(\mathrm{E})$ and relative Sun-Earth position is depicted, based on the global 251,107 shallow-source earthquakes $(4.0 \leq$ magnitude $<10.0$, focal depth $\leq 100 \mathrm{~km})$ from the 14 th to the $23 \mathrm{rd}$ solar 
The Characteristics of Global Shallow-Source Seismicities Associated with Solar Activities in Different Time Scales.

cycle (1902-2008). Blue lines: earthquakes frequency. Red lines: energy released from earthquakes.

\subsection{One-day Scale}

In order to search for the relationship between occurrence rates (N), energy released (E) from earthquakes and local time of the epicentral area, we analyses the global 251,107 shallowsource earthquakes $(4.0 \leqslant$ magnitude $<10.0$, focal depth $\leqslant 100 \mathrm{~km}$ ) from the 14 th to the 23rd solar cycle (1902-2008) in Figure 6. We find that earthquakes occur higher around $2 \sim 3$ am for the earthquakes $(4 \leqslant \mathrm{M}<8$ ), while around $4 \sim 5 \mathrm{am}, 1 \sim 2 \mathrm{pm}$ and $8 \sim 9 \mathrm{pm}$ for the earthquakes $(8 \leqslant \mathrm{M}<10)$. The largest released energy happened around $1 \sim 2$ am for the earthquakes $(8 \leqslant \mathrm{M}$ $<10)$.

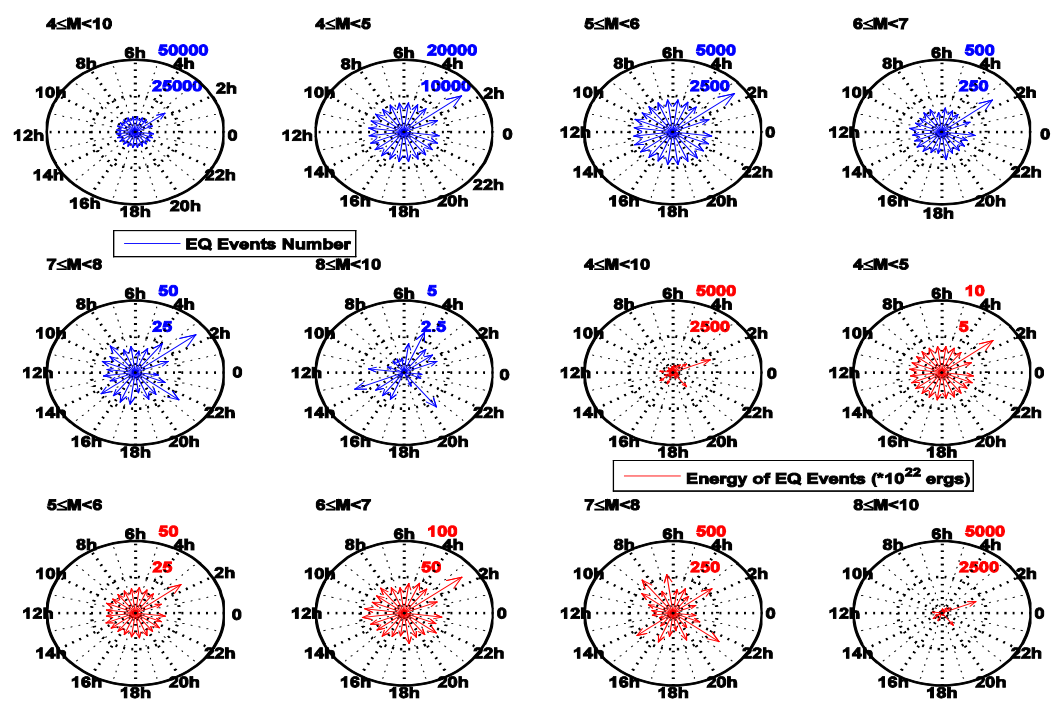

Figure 6. Relationship between occurrence frequency $(\mathrm{N})$, energy (E) released from earthquakes and local time of the epicenter, based on the global 251,107 shallow-source earthquakes ( $4.0 \leq$ magnitude $<10.0$, focal depth $\leq 100 \mathrm{~km}$ ) from the 14 th to the 23rd solar cycle (1902-2008). Blue lines: earthquakes frequency (N). Red lines: energy released from earthquakes (E).

\section{Discussion and Conclusions}

Solar activities can cause disturbance in the ionosphere $\mathrm{E}$ area which increase the turbulent state of D area, and it can lead to Kelvin Helmholtz instability. Kelvin - Helmholtz instabilities can interact with the local magnetic field lines, and motivate intermittent magnetic pulse which induces the earth current in the vast majority of susceptible area. As a solid electrolyte, fault zone can greatly increase the process of micro cracks, and weaken the earthquake zone to the bursting point.

In spite of the fact that the characteristics of seismicities assoctiated with solar activity is complex, we can still draw the following conclusions.

i) In century time scale, time series of sunspot numbers for the period (1700-2008) have obvious periodicity (about 11 years) by two different power spectral analysis methods (e.g. FFT algorithm and Lomb-Scargle algorithm). By using Lomb-Scargle spectrum analyses, the same period (about 11 years) also exits in the number of earthquakes $(4 \leqslant \mathrm{M}<5)$.

ii ) In decadal time scale, higher number of earthquakes occurred around descending phases of solar cycle than ascending phases. Released energy is the same. Furthermore, higher number and released energy from earthquakes occurred around one year before and after 
The Characteristics of Global Shallow-Source Seismicities Associated with Solar Activities in Different Time Scales.

minimum year $(\mathrm{m} \pm 1)$ and maximum year $(\mathrm{M} \pm 1)$ of sunspots numbers. The magnitude of an earthquake is higher, the more obvious trend.

iii) In one-year time scale, considering the perihelion (January 1st to January 5th) and the aphelion (July 3rd to July 4th), occurrence rates of earthquakes have no significant difference in every month. But the number and energy from earthquakes $(4 \leqslant \mathrm{M}<6)$ in February is less than the other months. For large earthquakes $(8 \leqslant \mathrm{M}<10)$ occurrence frequency $(\mathrm{N})$ are larger in March, August and October, and released energy (E) from earthquakes are more powerful in March and December. The energy released from shallow-source earthquakes with magnitude $(8 \sim 10)$ is dominant in the total seismic energy.

iv) In one-day time scale, earthquakes occur higher around $2 \sim 3$ am for the earthquakes $(4 \leqslant \mathrm{M}<8$ ), while around $4 \sim 5 \mathrm{am}, 1 \sim 2 \mathrm{pm}$ and $8 \sim 9 \mathrm{pm}$ for the earthquakes $(8 \leqslant \mathrm{M}<10)$. The largest released energy happened around $1 \sim 2$ am for the earthquakes $(8 \leqslant \mathrm{M}<10)$.

\section{References}

[1] Arcangelis, L. de., Lippiello, E., Godano, C., Nicodemi, M. 2008. Statistical properties and universality in earthquake and solar flare occurrence. Eur. Phys. J. B, 64, 551-555

[2] Arcangelis, L. de., Godano, C., Lippiello, E., Nicodemi, M. 2006. Universality in solar flare and earthquake occurrence. Phys. Rev. Lett, 96051102

[3] Balasis, G. et al. 2011.Universality in solar flare, magnetic storm and earthquake dynamics using Tsallis statistical mechanics. Physica A 390, 341-346

[4] Gleissberg, W. 1939. A long-periodic fluctuation of the sun-spot numbers. The Observatory 62, 158-159

[5] Gousheva, M. N., Georgiva, K.Y., Kirov, B.B., Atanssov, D. 2003. On the relation between solar activity and seismicity. Proceedings of International Conference on Recent Advances in Space Technologies November , 20-22

[6] Inan,U.S., Piddtachiy, D., Peter, W.B., Sauvaud, J.A., Parrot, M. 2007. Demeter satellite observations of lightning-induced electron precipitation. Geophys. Res. Lett 34, L07103

[7] Jusoh, M. H., Kiyohumi, Y. et al. 2011. Possible correlation between solar activity and global seismicity. Proceeding of the 2011 IEEE International Conference on Space Science and Communication (IconSpace)

[8] Kanamori, B.H. 1977. The energy release in great earthquakes. J. Geophys. Res. 82(20), 2981-2987

[9] Li, A. F. et al. 2008. Observation of periodic variation of cosmic ray intensity with the Tibet III air shower array. Nuclear Physics B (Proc. Suppl.) 175-176, 529-532

[10] Marilia, T., Anibal, A. 2011. Influence of solar cycles on earthquakes. Natural Science 3(6), 436-443

[11] Odintsov, S., Boyarchuk, K., Georgieva, K., Kirov, B., Atanasov, D. 2006. Long-period trends in global seismic and geomagnetic activity and their relation to solar activity. Physics and Chemistry of the Earth. 31, 88-93

[12] Scargle, J. D. 1982. Studies in astronomical time series analysis. II - statistical aspects of spectral analysis of unevenly spaced data. ApJ 263, 835-853

[13]Shaltout, M.A.M., Tadros, M.T.Y., Mesiha, S.L. 1999. Power spectra analysis for world-wide and North Africa historical earthquakes data in relation to sunspots periodicities. Renewable Energy 17, 499-507

[14] Simpson, J. F. 1967. Solar activity as a triggering mechanism for earthquakes. Earth and Planetary Science Letters 3, 417-425

[15]Yu, X. X., Lu, H., Shi, F. 2010. Influence of magnetic clouds on variation of cosmic rays in November 2004. Solar Physics 263, 223-237 